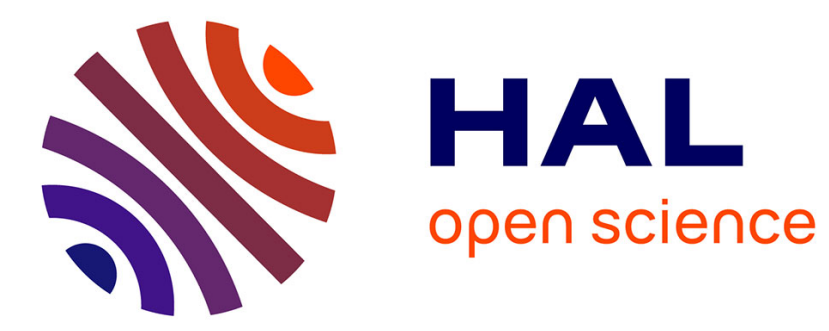

\title{
On necessary and sufficient conditions for output finite-time stability
}

Konstantin Zimenko, Denis Efimov, Andrey Polyakov, Artem Kremlev

\section{To cite this version:}

Konstantin Zimenko, Denis Efimov, Andrey Polyakov, Artem Kremlev. On necessary and sufficient conditions for output finite-time stability. Automatica, In press, 10.1016/j.automatica.2020.109427 . hal-02979811

\section{HAL Id: hal-02979811 \\ https://inria.hal.science/hal-02979811}

Submitted on 27 Oct 2020

HAL is a multi-disciplinary open access archive for the deposit and dissemination of scientific research documents, whether they are published or not. The documents may come from teaching and research institutions in France or abroad, or from public or private research centers.
L'archive ouverte pluridisciplinaire HAL, est destinée au dépôt et à la diffusion de documents scientifiques de niveau recherche, publiés ou non, émanant des établissements d'enseignement et de recherche français ou étrangers, des laboratoires publics ou privés. 


\title{
On necessary and sufficient conditions for output finite-time stability ${ }^{\star}$
}

\author{
Konstantin Zimenko a, Denis Efimov ${ }^{\text {b,a }}$, Andrey Polyakov ${ }^{\mathrm{b}, \mathrm{a}}$, Artem Kremlev ${ }^{\mathrm{a}}$ \\ ${ }^{a}$ Faculty of Control Systems and Robotics, ITMO University, 49 Kronverkskiy av., 197101 Saint Petersburg, Russia \\ ${ }^{\mathrm{b}}$ Inria, Univ. Lille, CNRS, UMR 9189 - CRIStAL, F-59000 Lille, France
}

\begin{abstract}
Output global finite-time stability of locally Lipschitz continuous autonomous systems is characterized by means of smooth Lyapunov functions. The so-called output-Lagrange stable systems are studied with details. Influence of a kind of continuity of the settling-time function is considered. Necessary and sufficient conditions of output finite-time stability are presented. The theoretical results are supported by academic examples and numerical simulations.
\end{abstract}

\section{Introduction}

Output stability addresses questions, where one is only concerned with stability and stabilizability of the output instead of the full set of state variables, i.e. an output variable $y(t) \rightarrow 0$ as $t \rightarrow \infty$. Such behavior is of great interest in control theory (see, for instance, [2]-[8], etc.). For example, the output stability analysis is required in a number of applications including both regulation (for instance, the use of adaptive control techniques ([11], [13], [14], [28], etc.), spacecraft stabilization [11], drift of the gyroscope axis [11], etc.) and observer design problems (for instance, where the output variables represent the observation error) [15], [16]. In addition, partial stability (see, for example, [9]-[19]) is a particular case of output stability, where the output is a subset of the state.

For locally Lipschitz continuous systems

$$
\dot{x}=f(x, u), \quad y=h(x),
$$

where $x, u$ and $y$ are the state, the input and the out-

\footnotetext{
* This work was partially supported by the Government of Russian Federation (Grant 08-08) and by the Ministry of Science and Higher Education of Russian Federation, passport of goszadanie no. 2019-0898.

Corresponding author K. Zimenko.

${ }^{\star \star}$ The material in this paper was partially presented at the 2019 European Control Conference [1].

Email addresses: kostyazimenko@gmail.com (Konstantin Zimenko), denis.efimov@inria.fr (Denis Efimov), andrey.polyakov@inria.fr (Andrey Polyakov), kremlev_artem@mail.ru (Artem Kremlev).
}

put, respectively, the papers [20]-[25] develop the theory of Input-to-Output Stability (IOS), that is immersed in the framework of Input-to-State Stability. The notion of IOS is devoted to robust output stability with respect to disturbances (inputs) $u$. In addition, the papers [22], [23] provide the results on output uniform global asymptotic stability (oUGAS) with respect to inputs from an admissible set $\mathcal{U}$ (or uniform output stability (UOS) in some references), i.e. the system output $y(t)$ tends to 0 as $t \rightarrow \infty$ for any $u$ from $\mathcal{U}$. Note, that this framework is given in terms of necessary and sufficient Lyapunov characterizations.

The present paper is devoted to the concept of Output Finite-Time Stability (OFTS) for autonomous systems. This notion implies completion of output transients in a finite time, i.e. the output $y(t)=0$ for all $t \geq T_{0}$ and some $0 \leq T_{0}<+\infty$. Such a system behaviour is needed in many control applications including mechanical and robotic systems, aerospace applications, particle collision systems, state and parameters observation, and adaptive finite-time control schemes (see, for example, [35], [34], [29], [30] [19], [18], etc.).

Note, that despite the name similarity of OFTS (or IOS) and Input-Output Finite-Time Stability (IO-FTS) (as, for example, presented in [27]), these notions are independent concepts, since the latter one implies that the output does not exceed an assigned threshold during a specified time interval.

The results presented in this paper are related to the class of systems that are locally Lipschitz out of the set $\mathcal{Y}=\left\{x \in \mathbb{R}^{n}: h(x)=0\right\}$ and continuous everywhere. 
Since this class is wider than considered in [22], [23], firstly, we extended the results on necessary and sufficient Lyapunov characterizations of oGAS for the system under consideration. Afterwards, for the first time, the necessary and sufficient conditions for OFTS of continuous autonomous systems have been derived taking into account the continuity of the settling-time function. It is noteworthy that in some cases an OFTS-Lyapunov function can be chosen in less restrictive form in comparison with Lyapunov functions presented in [22], [23].

Note, that most of existing results devoted to OFTS analysis are about partial stability conditions (a particular case of output stability) [10], [18], [19]. In addition, these conditions are rather restrictive for application. In this sense the necessary and sufficient conditions may simplify OFTS analysis for a wider class of systems and can be utilized for observation and control design problems.

Notation: $\mathbb{R}_{>0}=\{x \in \mathbb{R}: x>0\}, \mathbb{R}_{>0}=\{x \in \mathbb{R}: x \geq 0\}$, where $\mathbb{R}$ is the field of real numbers, $\mathbb{\mathbb { N }}$ is the set of natural numbers. $|\cdot|$ denotes the absolute value in $\mathbb{R},\|\cdot\|$ denotes the Euclidean norm on $\mathbb{R}^{n}$ (for $n \in \mathbb{N}$ ). A continuous function $\alpha: \mathbb{R}_{\geq 0} \rightarrow \mathbb{R}_{\geq 0}$ belongs to the class $\mathcal{K}$ if $\alpha(0)=$ 0 and the function is strictly increasing. The function $\alpha: \mathbb{R}_{\geq 0} \rightarrow \mathbb{R}_{\geq 0}$ belongs to the class $\mathcal{K}_{\infty}$ if $\alpha \in \mathcal{K}$ and it increases to infinity. A continuous function $\beta: \mathbb{R}_{\geq 0} \times$ $\mathbb{R}_{\geq 0} \rightarrow \mathbb{R}_{\geq 0}$ belongs to the class $\mathcal{K} \mathcal{L}$ if $\beta(\cdot, t) \in \mathcal{K}_{\infty}$ for each fixed $t \in \mathbb{R}_{>0}, \beta(s, \cdot)$ is decreasing and $\lim _{t \rightarrow+\infty} \beta(s, t)=$ 0 for each fixed $s \in \mathbb{R}_{\geq 0}$. For any real number $\alpha \in \mathbb{R}_{\geq 0}$ and for all real $x$ we set $\lfloor x\rceil^{\alpha}=\operatorname{sign}(x)|x|^{\alpha}$.

\section{Preliminaries}

\subsection{System under Consideration}

Consider the following nonlinear system:

$$
\dot{x}(t)=f(x(t)), \quad y(t)=h(x(t)), \quad t \geq 0,
$$

with states $x \in \mathbb{R}^{n}$ and outputs $y \in \mathbb{R}^{p}$.

Assumption 1 The vector field $f: \mathbb{R}^{n} \rightarrow \mathbb{R}^{n}$ ensures forward existence and uniqueness of the system solutions at least locally in time, $f(0)=0$.

Assumption 2 The function $h: \mathbb{R}^{n} \rightarrow \mathbb{R}^{p}$ is continuously differentiable, $h(0)=0$ and there exists $\gamma \in \mathcal{K}$ such that $\|h(x)\| \leq \gamma(\|x\|)$ for all $x \in \mathbb{R}^{n}$.

Assumption 3 The vector field $f$ is continuous on $\mathbb{R}^{n}$ and locally Lipschitz continuous on $\mathbb{R}^{n} \backslash \mathcal{Y}$, where $\mathcal{Y}=$ $\left\{x \in \mathbb{R}^{n}: h(x)=0\right\}$.

For the initial conditions $x_{0} \in \mathbb{R}^{n}$, let $X\left(t, x_{0}\right)$ be a unique solution of the system (1) defined over an interval $\left[0, T_{s}\right)$ where it exists with some $T_{s}>0$ (the solutions are understood in the Carathéodory sense), $Y\left(t, x_{0}\right)=$ $h\left(X\left(t, x_{0}\right)\right)$. A set $\mathcal{A} \subset \mathbb{R}^{n}$ is called forward invariant for (1) if $x_{0} \in \mathcal{A}$ implies that $X\left(t, x_{0}\right) \in \mathcal{A}$ for all $t \geq 0$. Note that due to regularity requirements imposed on $h$, the set $\mathcal{Y}$ is a submanifold in $\mathbb{R}^{n}$.

For a locally Lipschitz continuous function $V: \mathbb{R}^{n} \rightarrow \mathbb{R}$ the generalized directional derivative at $x \in \mathbb{R}^{n}$ along the solutions of $(1)$, is defined by:

$$
\dot{V}(x)=\limsup _{\substack{y \rightarrow x \\ h \rightarrow 0^{+}}} \frac{V(y+h f(x))-V(y)}{h} .
$$

If $V$ is continuously differentiable at $x \in \mathbb{R}^{n}$, then $\dot{V}(x)=<\nabla V(x), f(x)>$.

\subsection{Output Stability}

The preliminaries in this subsection are based on theoretical framework of IOS and oUGAS given in [20]-[25].

Definition 1 [22], [21] A system is forward complete if each $x_{0} \in \mathbb{R}^{n}$ produces a solution $X\left(t, x_{0}\right)$ which is defined for $t \in[0, \infty)$.

Definition 2 [20] The system (1) has the unboundedness observability $(U O)$ property if $\limsup _{t \rightarrow T_{s}}\left\|Y\left(t, x_{0}\right)\right\|=$ $+\infty$ necessarily follows for each $x_{0} \in \mathbb{R}^{n}$ such that $T_{s}<+\infty$.

In other words, any unboundedness of the state vector can be observed using the output $y$. Hence, if the output is known to be bounded (which is the case under the output stability properties described below) then the UO property is equivalent to forward completeness [22].

Definition 3 [23], [24] A system (1) is output globally asymptotically stable (oGAS) if it is forward complete, and there exists a $\mathcal{K} \mathcal{L}$-function $\beta$ such that

$$
\left\|Y\left(t, x_{0}\right)\right\| \leq \beta\left(\left\|x_{0}\right\|, t\right) \quad \forall t \geq 0
$$

holds for all $x_{0} \in \mathbb{R}^{n}$. If, in addition, there exists $\sigma \in \mathcal{K}$ such that

$$
\left\|Y\left(t, x_{0}\right)\right\| \leq \sigma\left(\left\|h\left(x_{0}\right)\right\|\right), \quad \forall t \geq 0
$$

holds for all trajectories of the system, then the system is output-Lagrange output globally asymptotically stable (OLoGAS). Finally, if one strengthens (2) to

$$
\left\|Y\left(t, x_{0}\right)\right\| \leq \beta\left(\left\|h\left(x_{0}\right)\right\|, t\right), \quad \forall t \geq 0
$$

holding for all trajectories of the system, then the system is state-independent output globally asymptotically stable (SIoGAS). 
The following implications is a direct consequence of Definition 3 [25]:

$$
S I o G A S \Rightarrow O L o G A S \Rightarrow o G A S
$$

Let us present definitions for corresponding Lyapunov functions.

Definition 4 [22] [23] For the system (1), a smooth function $V$ and a function $\lambda: \mathbb{R}^{n} \rightarrow \mathbb{R}_{>0}$ are called respectively an oGAS-Lyapunov function and an auxiliary modulus if there exist $\alpha_{1}, \alpha_{2} \in \mathcal{K}_{\infty}$ so that

$$
\alpha_{1}(\|h(x)\|) \leq V(x) \leq \alpha_{2}(\|x\|) \quad \forall x \in \mathbb{R}^{n}
$$

holds and there exists $\alpha_{3} \in \mathcal{K} \mathcal{L}$ such that

$$
\dot{V}(x) \leq-\alpha_{3}(V(x), \lambda(x))
$$

for all $x \in \mathcal{X}$, where $\mathcal{X}=\left\{x \in \mathbb{R}^{n}: V(x)>0\right\}$, and either

(a) $\lambda$ satisfies the following conditions:

- $0 \leq \lambda(x) \leq\|x\|$ for all $x \in \mathbb{R}^{n}$;

- $\lambda$ is locally Lipschitz on the set $\mathcal{X}$ and satisfies

$$
D \lambda(x) f(x) \leq 0 \quad \text { for a.a. } x \in \mathcal{X}
$$

or

(b) there exists some $\theta \in \mathcal{K}$ such that

$$
\lambda\left(X\left(t, x_{0}\right)\right) \leq \theta\left(\left\|x_{0}\right\|\right) \quad \text { for all } t \in \mathbb{R}_{\geq 0}, x_{0} \in \mathcal{X}
$$

The function $V$ is called an OLoGAS-Lyapunov function if it is an oGAS-Lyapunov function, and in addition, inequality (5) can be strengthened to

$$
\alpha_{1}(\|h(x)\|) \leq V(x) \leq \alpha_{2}(\|h(x)\|), \quad \forall x \in \mathbb{R}^{n} .
$$

The function $V$ is called a SIoGAS-Lyapunov function if the inequality (9) is satisfied and there exists $\alpha_{3} \in \mathcal{K}$ such that for all $x \in \mathcal{X}$ :

$$
\dot{V}(x) \leq-\alpha_{3}(V(x)) .
$$

An auxiliary modulus $\lambda$ satisfying property (a) is called a strong auxiliary modulus, and one satisfying property (b) is a weak auxiliary modulus [22].

Note that in the case of OLoGAS- or SIoGAS-Lyapunov function we have $\mathcal{X}=\mathbb{R}^{n} \backslash \mathcal{Y}$. The difference between the oGAS and OLoGAS/SIoGAS properties becomes more evident by comparing the corresponding Lyapunov functions. For OLoGAS/SIoGAS, the Lyapunov function is bounded from above by the function of distance to the set $\mathcal{Y}$, whereas for the oGAS system the Lyapunov function can grow proportionally to $\|x\|$.
Above definitions (and an analogue of Theorem 1 given below) are presented in [20]-[25] for the dynamical system $\dot{x}=f(x, u), y=h(x)$ with locally Lipschitz continuous $f, h$ in the sense of uniform stability with respect to inputs $u$. Despite this, all these definitions remain valid for the class of systems under consideration. In [22], [23] it has been show that existence of a Lyapunov function given in Definition 4 is equivalent to a respective stability property from Definition 3 (the case of bounded-inputbounded-state (BIBS) systems was studied in [23]).

\subsection{Output Finite-Time Stability}

Now let us present the definition on OFTS:

Definition 5 The system (1) is said to be OFTS if it is oGAS and for any $x_{0} \in \mathbb{R}^{n}$ there exists $0 \leq T_{0}<$ $+\infty$ such that $Y\left(t, x_{0}\right)=0$ for all $t>T_{0}$. The function $T\left(x_{0}\right)=\inf \left\{T_{0} \geq 0: Y\left(t, x_{0}\right)=0 \quad \forall t \geq T_{0}\right\}$ is called the settling-time function.

Under imposed assumptions on $f$ the solution of $(1)$ defines a continuous global semiflow. Then $X: \mathbb{R}_{\geq 0} \times$ $\mathbb{R}^{n} \rightarrow \mathbb{R}^{n}$ is a (jointly) continuous function satisfying $X\left(0, x_{0}\right)=x_{0}, X\left(t, X\left(\tau, x_{0}\right)\right)=X\left(t+\tau, x_{0}\right)$ and we have

$$
Y\left(0, x_{0}\right)=h\left(x_{0}\right)
$$

$$
\begin{gathered}
Y\left(t, X\left(\tau, x_{0}\right)\right)=Y\left(t+\tau, x_{0}\right), \\
Y\left(T\left(x_{0}\right)+t, x_{0}\right)=0,
\end{gathered}
$$

where $t, \tau \in \mathbb{R}_{\geq 0}, x_{0} \in \mathbb{R}^{n}$.

\subsection{Lemmas regarding $\mathcal{K}$ and $\mathcal{K} \mathcal{L}$ functions}

Let us give supporting lemmas that are used through the paper.

Lemma 1 [31] For any $\alpha \in \mathcal{K}$ there exists a continuous function $\gamma: \mathbb{R}_{\geq 0} \rightarrow \mathbb{R}_{\geq 0}$ admitting the following properties: $\gamma(0)=0, \gamma(s)>0$ for all $s>0$, and

$$
\gamma(s) \leq \alpha(s),\left|\gamma(s)-\gamma\left(s^{\prime}\right)\right| \leq\left|s-s^{\prime}\right| \quad \forall s, s^{\prime} \in \mathbb{R}_{\geq 0} .
$$

In addition, $\lim _{s \rightarrow+\infty} \gamma(s)=+\infty$ provided that $\alpha \in \mathcal{K}_{\infty}$.

An expression for such a function is

$$
\gamma(s)=\min _{0 \leq y \leq s}\{\alpha(y)+s-y\}
$$

Lemma 2 [32] For any $\beta \in \mathcal{K} \mathcal{L}$ there exist $\theta_{1}, \theta_{2} \in \mathcal{K}_{\infty}$ such that $\beta(s, t) \leq \theta_{1}\left(\theta_{2}(s) e^{-t}\right)$ for all $s \geq 0, t \geq 0$. 


\section{Main result}

In this section we will assume that assumptions 1-3 are satified, then the system under consideration (1) is of a wider class than in [22] and [23] (the Lipschitz continuity may be violated on $\mathcal{Y}$ ). To give the necessary and sufficient conditions for OFTS, let us first extend results of [22], [23] and give the necessary and sufficient Lyapunov characterizations of output stability for the system (1).

Theorem 1 Let the system (1) be UO and assumptions 1-3 be satisfied.

(i) The system is OLoGAS if and only if there is an OLoGAS-Lyapunov function.

(ii) The system is SIoGAS if and only if there is a SIoGASLyapunov function.

Proof. Sufficiency. The sufficiency follows from [22].

Necessity. (i) For brevity the proof is given for the case of $\lambda(x)=\|x\|$ (i.e., the BIBS case as in [23]), and the results with strong or weak auxiliary modulus can be obtained as in [22].

Under the introduced hypothesis and Lemma 2 there are $\theta_{1}, \theta_{2}, \sigma \in \mathcal{K}_{\infty}$ such that

$$
\left\|Y\left(t, x_{0}\right)\right\| \leq \min \left\{\sigma\left(\left\|h\left(x_{0}\right)\right\|\right), \theta_{1}\left(\theta_{2}\left(\left\|x_{0}\right\|\right) e^{-t}\right)\right\} \quad \forall t \geq 0
$$

for all $x_{0} \in \mathbb{R}^{n}$. Recalling Lemma 1, there exists a continuous, positive definite and unbounded function $\gamma: \mathbb{R}_{\geq 0} \rightarrow \mathbb{R}_{\geq 0}$ admitting the following properties:

$\gamma(s) \leq \min \left\{s, \theta_{1}^{-1}(s)\right\},\left|\gamma(s)-\gamma\left(s^{\prime}\right)\right| \leq\left|s-s^{\prime}\right| \forall s, s^{\prime} \in \mathbb{R}_{\geq 0}$.

Next, for any $x_{0} \in \mathbb{R}^{n}$ select

$$
V\left(x_{0}\right)=\sup _{t \geq 0}\left\{\gamma\left(\left\|Y\left(t, x_{0}\right)\right\|\right) \frac{\kappa_{1}+t}{\kappa_{2}+t}\right\}
$$

with $\frac{\kappa_{2}}{1+\kappa_{2}}<\kappa_{1}<\kappa_{2}<+\infty$. Then

$$
V\left(x_{0}\right) \leq \sup _{t \geq 0}\left\{\sigma\left(\left\|h\left(x_{0}\right)\right\|\right) \frac{\kappa_{1}+t}{\kappa_{2}+t}\right\} \leq \sigma\left(\left\|h\left(x_{0}\right)\right\|\right)
$$

and

$$
V\left(x_{0}\right) \geq \sup _{t \geq 0}\left\{\gamma\left(\left\|Y\left(t, x_{0}\right)\right\|\right)\right\} \frac{\kappa_{1}}{\kappa_{2}} \geq \gamma\left(\left\|h\left(x_{0}\right)\right\|\right) \frac{\kappa_{1}}{\kappa_{2}}
$$

since under introduced restrictions on $\kappa_{1}$ and $\kappa_{2}$ the function $\frac{\kappa_{1}+t}{\kappa_{2}+t}$ is strictly increasing $\left(\frac{\kappa_{1}}{\kappa_{2}} \leq \frac{\kappa_{1}+t}{\kappa_{2}+t} \leq 1\right.$ for all $t \geq 0)$. Note that from this analysis $\frac{\kappa_{1}}{\kappa_{2}} \gamma\left(\left\|h\left(x_{0}\right)\right\|\right) \leq$ $\min \left\{\sigma\left(\left\|h\left(x_{0}\right)\right\|\right), \theta_{2}\left(\left\|x_{0}\right\|\right)\right\}$ for all $x_{0} \in \mathbb{R}^{n}$. Define $\gamma(s)=$ $\frac{s}{1+s} \inf _{r \geq s} \gamma(r)$, which is a $\mathcal{K}_{\infty}$ class function, then

$$
\frac{\kappa_{1}}{\kappa_{2}} \underline{\gamma}\left(\left\|h\left(x_{0}\right)\right\|\right) \leq V\left(x_{0}\right) \leq \sigma\left(\left\|h\left(x_{0}\right)\right\|\right)
$$

for all $x_{0} \in \mathbb{R}^{n}$. Under introduced restrictions on $\kappa_{1}$ and $\kappa_{2}$ the function $e^{-t} \frac{\kappa_{1}+t}{\kappa_{2}+t}$ is strictly decreasing, then there exists $T^{x_{0}}>0$ such that

$$
\begin{aligned}
& V\left(x_{0}\right)=\sup _{0 \leq t \leq T^{x_{0}}}\left\{\gamma\left(\left\|Y\left(t, x_{0}\right)\right\|\right) \frac{\kappa_{1}+t}{\kappa_{2}+t}\right\} \\
& =\gamma\left(\left\|Y\left(T^{x_{0}}, x_{0}\right)\right\|\right) \frac{\kappa_{1}+T^{x_{0}}}{\kappa_{2}+T^{x_{0}}} \leq \theta_{2}\left(\left\|x_{0}\right\|\right) e^{-T^{x_{0}}}
\end{aligned}
$$

and since

$$
\gamma\left(\left\|h\left(x_{0}\right)\right\|\right) \frac{\kappa_{1}}{\kappa_{2}} \leq V\left(x_{0}\right) \leq \theta_{2}\left(\left\|x_{0}\right\|\right) e^{-T^{x_{0}}},
$$

then by the definition of $T^{x_{0}}$ it has an upper estimate:

$$
T^{x_{0}} \leq \ln \left[\frac{\kappa_{2}}{\kappa_{1}} \frac{\theta_{2}\left(\left\|x_{0}\right\|\right)}{\gamma\left(\left\|h\left(x_{0}\right)\right\|\right)}\right],
$$

where we recall that $\frac{\kappa_{1}}{\kappa_{2}} \gamma\left(\left\|h\left(x_{0}\right)\right\|\right) \leq \theta_{2}\left(\left\|x_{0}\right\|\right)$ for all $x_{0} \in \mathbb{R}^{n}$ and the left-hand side of the above inequality is always positive. Hence on any compact subset of $\mathbb{R}^{n} \backslash \mathcal{Y}$ there exists a finite upper bound on $T^{x_{0}}$

Let us check the local Lipschitz continuity of $V$ on $\mathbb{R}^{n} \backslash \mathcal{Y}$. To this end, for any $x_{0} \in \mathbb{R}^{n} \backslash \mathcal{Y}$ select its compact vicinity $\Omega \subset \mathbb{R}^{n} \backslash \mathcal{Y}, x_{0} \in \Omega$. Denote $T^{\Omega}=\sup _{x_{0} \in \Omega} T^{x_{0}}$, and assume that the set $\Omega$ is chosen in a way that

$$
\inf _{t \in\left[0, T^{\Omega}\right], x_{0} \in \Omega}\left\|Y\left(t, x_{0}\right)\right\|>0
$$

it is always possible due to continuous dependence of the solutions on initial conditions in $\mathbb{R}^{n} \backslash \mathcal{Y}$ and the definition of $T^{x_{0}}$. Take any $x_{1} \in \Omega$, then consider:

$$
\begin{aligned}
& \left|V\left(x_{1}\right)-V\left(x_{0}\right)\right|=\mid \sup _{0 \leq t \leq T^{x_{1}}}\left\{\gamma\left(\left\|Y\left(t, x_{1}\right)\right\|\right) \frac{\kappa_{1}+t}{\kappa_{2}+t}\right\} \\
& \quad-\sup _{0 \leq t \leq T^{x_{0}}}\left\{\gamma\left(\left\|Y\left(t, x_{0}\right)\right\|\right) \frac{\kappa_{1}+t}{\kappa_{2}+t}\right\} \mid \\
& \leq \sup _{0 \leq t \leq T^{\Omega}}\left|\left[\gamma\left(\left\|Y\left(t, x_{1}\right)\right\|\right)-\gamma\left(\left\|Y\left(t, x_{0}\right)\right\|\right)\right] \frac{\kappa_{1}+t}{\kappa_{2}+t}\right| \\
& \leq \sup _{0 \leq t \leq T^{\Omega}}\left|\gamma\left(\left\|Y\left(t, x_{1}\right)\right\|\right)-\gamma\left(\left\|Y\left(t, x_{0}\right)\right\|\right)\right| \\
& \leq \sup _{0 \leq t \leq T^{\Omega}}\left|\left\|h\left(X\left(t, x_{1}\right)\right)\right\|-\left\|h\left(X\left(t, x_{0}\right)\right)\right\|\right| \\
& \leq \sup _{0 \leq t \leq T^{\Omega}}\left\|h\left(X\left(t, x_{1}\right)\right)-h\left(X\left(t, x_{0}\right)\right)\right\|,
\end{aligned}
$$

where on the last step and the step before the global Lipschitz properties of the norm $\|\cdot\|$ and the function $\gamma$ have been utilized, respectively. Due to Lipschitz continuity 
of the system on the set $\mathbb{R}^{n} \backslash \mathcal{Y}$ here exists $M_{T^{\Omega}, \Omega}>0$ such that

$$
\left\|h\left(X\left(t, x_{1}\right)\right)-h\left(X\left(t, x_{0}\right)\right)\right\| \leq M_{T^{\Omega}, \Omega}\left\|x_{1}-x_{0}\right\|
$$

for all $t \in\left[0, T^{\Omega}\right]$ since $h$ is continuously differentiable and $\left\|h\left(X\left(t, x_{i}\right)\right)\right\|$ is bounded for all $t \geq 0$ and $i=0,1$. Hence, $\left|V\left(x_{1}\right)-V\left(x_{0}\right)\right| \leq M_{T^{\Omega}, \Omega}\left\|x_{1}-x_{0}\right\|$ for all such $x_{0}, x_{1} \in \Omega$, which implies the Lipschitz continuity of $V(x)$ in a vicinity of any $x \in \mathbb{R}^{n} \backslash \mathcal{Y}$, which is equivalent for $V$ to be locally Lipschitz continuous in $\mathbb{R}^{n} \backslash \mathcal{Y}$. The continuity at $\mathcal{Y}$ can be obtained from the upper estimate $V\left(x_{0}\right) \leq \sigma\left(\left\|h\left(x_{0}\right)\right\|\right)$ that is satisfied for all $x_{0} \in \mathbb{R}^{n}$.

Finally, let us check the decreasing of the Lyapunov function $V$ on the trajectories of the system (1) with $x_{0} \in \mathbb{R}^{n} \backslash \mathcal{Y}$ for $t>0$ (recall that $\frac{\kappa_{1}+t}{\kappa_{2}+t}$ is a strictly increasing function of time):

$$
\begin{aligned}
& V\left(X\left(t, x_{0}\right)\right)=\sup _{r \geq 0}\left\{\gamma\left(\left\|Y\left(r, X\left(t, x_{0}\right)\right)\right\|\right) \frac{\kappa_{1}+r}{\kappa_{2}+r}\right\} \\
& =\sup _{r \in\left[0, T^{X\left(t, x_{0}\right)}\right]}\left\{\gamma\left(\left\|Y\left(r, X\left(t, x_{0}\right)\right)\right\|\right) \frac{\kappa_{1}+r}{\kappa_{2}+r}\right\} \\
& =\sup _{r \in\left[0, T^{X\left(t, x_{0}\right)}\right]}\left\{\gamma\left(\left\|Y\left(t+r, x_{0}\right)\right\|\right) \frac{\kappa_{1}+r}{\kappa_{2}+r}\right\} \\
& =\sup _{r \in\left[t, t+T^{X\left(t, x_{0}\right)}\right]}\left\{\gamma\left(\left\|Y\left(r, x_{0}\right)\right\|\right) \frac{\kappa_{1}+r-t}{\kappa_{2}+r-t}\right\} \\
& \leq \sup _{r \in\left[t, t+T^{X\left(t, x_{0}\right)}\right]}\left\{\gamma\left(\left\|Y\left(r, x_{0}\right)\right\|\right) \frac{\kappa_{1}+r}{\kappa_{2}+r}\right\} \\
& +\sup _{r \in\left[t, t+T^{X\left(t, x_{0}\right)}\right]}\left\{\gamma\left(\left\|Y\left(r, x_{0}\right)\right\|\right)\left(\frac{\kappa_{1}+r-t}{\kappa_{2}+r-t}-\frac{\kappa_{1}+r}{\kappa_{2}+r}\right)\right\} \\
& \leq \sup _{r \in\left[t, t+T^{X\left(t, x_{0}\right)}\right]}\left\{\gamma\left(\left\|Y\left(r, x_{0}\right)\right\|\right) \frac{\kappa_{1}+r}{\kappa_{2}+r}\right\} \\
& +\sigma\left(\left\|h\left(x_{0}\right)\right\|\right) \sup _{r \in\left[t, t+T^{X\left(t, x_{0}\right)}\right]}\left\{\frac{\kappa_{1}+r-t}{\kappa_{2}+r-t}-\frac{\kappa_{1}+r}{\kappa_{2}+r}\right\} \\
& \leq \sup _{r \geq 0}\left\{\gamma\left(\left\|Y\left(r, x_{0}\right)\right\|\right) \frac{\kappa_{1}+r}{\kappa_{2}+r}\right\} \\
& +\sigma\left(\left\|h\left(x_{0}\right)\right\|\right) \sup _{r \in\left[t, t+T^{X\left(t, x_{0}\right)}\right]}\left\{\frac{\kappa_{1}+r-t}{\kappa_{2}+r-t}-\frac{\kappa_{1}+r}{\kappa_{2}+r}\right\} \\
& =V\left(x_{0}\right)+\sigma\left(\left\|h\left(x_{0}\right)\right\|\right) \sup _{r \in\left[t, t+T^{X\left(t, x_{0}\right)}\right]}\left\{\frac{\kappa_{1}+r-t}{\kappa_{2}+r-t}-\frac{\kappa_{1}+r}{\kappa_{2}+r}\right\} \text {, }
\end{aligned}
$$

thus, $V$ is strictly decreasing along the trajectories of (1) since $\sup _{r \in\left[t, t+T^{X\left(t, x_{0}\right)}\right]}\left\{\frac{\kappa_{1}+r-t}{\kappa_{2}+r-t}-\frac{\kappa_{1}+r}{\kappa_{2}+r}\right\}<0$ for any $t>0$. Consequently, computing directional derivative of
$V(x)$ for $x \in \mathbb{R}^{n} \backslash \mathcal{Y}$ we get:

$$
\begin{gathered}
\dot{V}(x)=\limsup _{\substack{z \rightarrow x \\
h \rightarrow 0^{+}}} \frac{V(z+h f(x))-V(z)}{h} \\
=\limsup _{t \rightarrow 0} \frac{V(X(t, x))-V(x)}{t} \\
\leq \sigma(\|h(x)\|) \limsup _{t \rightarrow 0} \frac{\sup _{r \in\left[t, t+T^{X(t, x)}\right]\left\{\frac{\kappa_{1}+r-t}{\kappa_{2}+r-t}-\frac{\kappa_{1}+r}{\kappa_{2}+r}\right\}}}{t},
\end{gathered}
$$

where on the last step we use the upper bound for $V(X(t, x))-V(x)$ obtained above. Note that by the Mean Value Theorem:

$$
\frac{\kappa_{1}+r-t}{\kappa_{2}+r-t}-\frac{\kappa_{1}+r}{\kappa_{2}+r}=-t \frac{\kappa_{2}-\kappa_{1}}{\left(\theta+\kappa_{2}\right)^{2}},
$$

where $\theta \in[r-t, r]$, then

$$
\begin{gathered}
\sup _{r \in\left[t, t+T^{X(t, x)}\right]}\left\{\frac{\kappa_{1}+r-t}{\kappa_{2}+r-t}-\frac{\kappa_{1}+r}{\kappa_{2}+r}\right\} \\
=\sup _{r \in\left[t, t+T^{X(t, x)}\right]} \sup _{\theta \in[r-t, r]}\left\{-t \frac{\kappa_{2}-\kappa_{1}}{\left(\theta+\kappa_{2}\right)^{2}}\right\} \\
\leq-t \frac{\kappa_{2}-\kappa_{1}}{\left(t+T^{X(t, x)}+\kappa_{2}\right)^{2}} .
\end{gathered}
$$

Finally,

$$
\begin{aligned}
\dot{V}(x) & \leq-\sigma(\|h(x)\|) \limsup _{t \rightarrow 0} \frac{\kappa_{2}-\kappa_{1}}{\left(t+T^{X(t, x)}+\kappa_{2}\right)^{2}} \\
& =-\sigma(\|h(x)\|) \frac{\kappa_{2}-\kappa_{1}}{\left(T^{x}+\kappa_{2}\right)^{2}} \\
& \leq-\sigma(\|h(x)\|) \frac{\kappa_{2}-\kappa_{1}}{\left(\ln \left[\frac{\kappa_{2}}{\kappa_{1}} \frac{\theta_{2}(\|x\|)}{\gamma(\|h(x)\|)}\right]+\kappa_{2}\right)^{2}} \\
& \leq-\beta(V(x),\|x\|)
\end{aligned}
$$

for some $\beta \in \mathcal{K} \mathcal{L}$ and almost all $x \in \mathbb{R}^{n} \backslash \mathcal{Y}$ (recall again that $\frac{\kappa_{1}}{\kappa_{2}} \gamma(\|h(x)\|) \leq \theta_{2}(\|x\|)$ for all $\left.x \in \mathbb{R}^{n}\right)$. Since $V(x)=0$ for $x \in \mathcal{Y}$, then the above estimate holds almost globally. The obtained OLoGAS-Lyapunov function $V$ is locally Lipschitz continuous, next a smooth one can be obtained using the standard smoothing tools as in [33], [23].

(ii) Suppose that one strengthens (3) to (4). Since (14) can be strengthened to

$$
\begin{gathered}
\left\|Y\left(t, x_{0}\right)\right\| \leq \min \left\{\sigma\left(\left\|h\left(x_{0}\right)\right\|\right), \theta_{1}\left(\theta_{2}\left(\left\|h\left(x_{0}\right)\right\|\right) e^{-t}\right)\right\} \\
\forall t \geq 0,
\end{gathered}
$$

we now repeat the above proof to get a function $V(x)$ satisfying (9), and

$$
\dot{V}(x) \leq-\beta(V(x),\|h(x)\|) \leq-\beta(V(x), V(x))
$$


for almost all $x \in \mathbb{R}^{n} \backslash \mathcal{Y}$. Then, following to the proof of Theorem 3.2 in [23] we can show that there is a Lyapunov function $V_{1}$ such that (9) and (10) hold for some $\alpha_{1}, \alpha_{2} \in$ $\mathcal{K}_{\infty}$ and $\alpha_{3} \in \mathcal{K}$.

Based on given characterizations we may obtain the following results on OFTS.

Theorem 2 Consider the UO system (1). The following properties are equivalent:

(1) the system is OFTS satisfying the condition (3);

(2) there exists a smooth OLoGAS-Lyapunov function $V: \mathbb{R}^{n} \rightarrow \mathbb{R}_{\geq 0}$ for the system (1) satisfying for all $x \in \mathbb{R}^{n}$

$$
\int_{0}^{V(x)} \frac{d s}{-\dot{V}\left(X\left(\theta_{x}(s), x\right)\right)}<+\infty
$$

where $\theta_{x}$ is the inverse of $t \mapsto V(X(t, x))$.

Proof. 1. $\Rightarrow 2$. If the system (1) is output finite-time stable with the settling-time function $T: \mathbb{R}^{n} \rightarrow \mathbb{R}_{\geq 0}$ and (3) is satisfied, then according to Theorem 1 there exists a smooth OLoGAS-Lyapunov function $V: \mathbb{R}^{n} \rightarrow$ $\mathbb{R}_{\geq 0}$ for the system (1). Since the well-defined application $[0, T(x)) \rightarrow(0, V(x)], t \mapsto V(X(t, x))$ is strictly decreasing and differentiable, so its inverse $(0, V(x)] \rightarrow$ $[0, T(x)), s \mapsto \theta_{x}(s)$ is differentiable and satisfies for all $s \in(0, V(x)]$,

$$
\theta_{x}^{\prime}(s)=\frac{1}{\dot{V}\left(X\left(\theta_{x}(s), x\right)\right)}
$$

The change of variables $s=V(X(t, x))$ and the fact that $V(X(T(x), x))=0($ due to $(9))$ lead to

$$
\begin{gathered}
T(x)=\int_{0}^{T(x)} d t=\int_{V(x)}^{0} \theta_{x}^{\prime}(s) d s \\
=\int_{0}^{V(x)} \frac{d s}{-\dot{V}\left(X\left(\theta_{x}(s), x\right)\right)}<+\infty .
\end{gathered}
$$

2. $\Rightarrow 1$. As there exists an OLoGAS-Lyapunov function for the system (1), Theorem 1 ensures that it is output stable. The equality (16) implies the finite-time convergence of $V\left(X\left(t, x_{0}\right)\right)$ (as well as $\left.Y\left(t, x_{0}\right)\right)$ to zero.

According to [23], for any $\alpha_{3} \in \mathcal{K} \mathcal{L}$, there exist $\kappa_{1}, \kappa_{2} \in$ $\mathcal{K}$ such that $\alpha_{3}(s, t) \geq \frac{\kappa_{1}(s)}{1+\kappa_{2}(t)}$ for all $s, t \in \mathbb{R}_{\geq 0}$. Hence, for an OLoGAS-Lyapunov function with a strong auxiliary modulus $\lambda$ we have

$$
\dot{V}(x) \leq-\frac{\kappa_{1}(V(x))}{1+\kappa_{2}(\lambda(x))}
$$

and the following corollary to Theorem 2 on sufficient condition of OFTS can be given.
Corollary 1 Consider the OLoGAS system (1). Let $V$ be an OLoGAS-Lyapunov function satisfying the inequality

$$
\int_{0}^{V\left(x_{0}\right)} \frac{d s}{\kappa_{1}(s)}<+\infty,
$$

with $\kappa_{1} \in \mathcal{K}$ as in (17). Then the system (1) is OFTS.

Proof. Since the system is OLoGAS, then there exists OLoGAS-Lyapunov function with strong auxiliary modulus (see Theorem 1). Due to (7) we have $\lambda(x) \leq \lambda\left(x_{0}\right) \leq$ $\left\|x_{0}\right\|$. Then, taking into account that $V\left(X\left(\theta_{x}(\bar{s}), x\right)\right)=s$ by the definition of the map $\theta_{x}$ and using (17), (18), we have

$$
\begin{gathered}
T\left(x_{0}\right)=\int_{0}^{V\left(x_{0}\right)} \frac{d s}{-\dot{V}\left(X\left(\theta_{x}(s), x\right)\right)} \\
\leq \int_{0}^{V\left(x_{0}\right)} \frac{d s}{\alpha_{3}\left(V\left(X\left(\theta_{x}(s), x\right)\right), \lambda\left(X\left(\theta_{x}(s), x\right)\right)\right)} \\
\leq \int_{0}^{V\left(x_{0}\right)} \frac{d s}{\alpha_{3}\left(s,\left\|x_{0}\right\|\right)} \leq\left(1+\kappa_{2}\left(\left\|x_{0}\right\|\right)\right) \int_{0}^{V\left(x_{0}\right)} \frac{d s}{\kappa_{1}(s)}<+\infty .
\end{gathered}
$$

Finding an OLoGAS-Lyapunov function with a weak or strong auxiliary modulus is a difficult task in some cases. Let us develop those notions and give the definition for a local auxiliary modulus.

Definition 6 An auxiliary modulus $\lambda$ in (6) is called local auxiliary modulus if there exists some (jointly) continuous function $\varrho: \mathbb{R}_{\geq 0} \times \mathbb{R}_{\geq 0} \rightarrow \mathbb{R}_{\geq 0}$ such that $\varrho(\cdot, t)$ is nondecreasing, $\varrho(s, \cdot) \in \mathcal{K}$ and for any $T \in \mathbb{R}_{\geq 0}$

$$
\lambda\left(X\left(t, x_{0}\right)\right) \leq \varrho\left(\left\|x_{0}\right\|, T\right)
$$

for all $t \in[0, T]$ and $x_{0} \in \mathcal{X}$.

Using Definition 6 the following result can be given.

Lemma 3 Consider the UO system (1). Let there exist a smooth Lyapunov function $V: \mathbb{R}^{n} \rightarrow \mathbb{R}_{\geq 0}$ for the system (1) satisfying the conditions (6) and (9) with a local auxiliary modulus $\lambda$. If for any $\varepsilon \in \mathbb{R}_{\geq 0}$ there exists $T_{\varepsilon} \in \mathbb{R}_{\geq 0}$ such that

$$
\int_{0}^{\alpha_{2} \circ \gamma(\varepsilon)} \frac{d s}{\alpha_{3}\left(s, \varrho\left(\varepsilon, T_{\varepsilon}\right)\right)} \leq T_{\varepsilon}<+\infty
$$

then the system (1) is OFTS. Moreover, $T\left(x_{0}\right) \leq T_{\varepsilon}$ for $\left\{x_{0} \in \mathbb{R}^{n}:\left\|x_{0}\right\| \leq \varepsilon\right\}$.

Proof. For any $\varepsilon \in \mathbb{R}_{>0}$ and $x_{0} \in \mathbb{R}^{n}$ such that $\left\|x_{0}\right\| \leq \varepsilon$ due to (6) and (19) we have

$$
\begin{gathered}
\dot{V}\left(X\left(t, x_{0}\right)\right) \leq-\alpha_{3}\left(V\left(X\left(t, x_{0}\right)\right), \lambda\left(X\left(t, x_{0}\right)\right)\right) \\
\leq-\alpha_{3}\left(V\left(X\left(t, x_{0}\right)\right), \varrho\left(\left\|x_{0}\right\|, T\right)\right) \leq-\alpha_{3}(V(x), \varrho(\varepsilon, T))
\end{gathered}
$$


for all $t \in[0, T]$. Following the proof of Theorem 2 we have

$$
\begin{aligned}
& T(x)=\int_{0}^{V(x)} \frac{d s}{-\dot{V}\left(X\left(\theta_{x}(s), x\right)\right)} \\
\leq & \int_{0}^{V(x)} \frac{d s}{\alpha_{3}\left(V\left(X\left(\theta_{x}(s), x\right)\right), \varrho(\varepsilon, T(x))\right)} .
\end{aligned}
$$

Let $T_{\varepsilon} \geq T\left(x_{0}\right)$. Then, taking into account that $V\left(X\left(\theta_{x}(s), x\right)\right)=s$ by the definition of the map $\theta_{x}$ and using (21) and (22), we have

$$
\begin{aligned}
& T\left(x_{0}\right) \leq \int_{0}^{V\left(x_{0}\right)} \frac{d s}{\alpha_{3}\left(s, \varrho\left(\varepsilon, T\left(x_{0}\right)\right)\right)} \\
\leq & \int_{0}^{\alpha_{2} \circ \gamma(\varepsilon)} \frac{d s}{\alpha_{3}\left(s, \varrho\left(\varepsilon, T_{\varepsilon}\right)\right)} \leq T_{\varepsilon}<+\infty
\end{aligned}
$$

that proofs that $T\left(x_{0}\right) \leq T_{\varepsilon}$ and the system is OFTS.

Remark 1 Note that local auxiliary modulus is less restrictive than weak auxiliary modulus (8), that makes the search for the OFTS-Lyapunov function (9), (6), (19) even simpler in comparison with the OLoGAS-Lyapunov function presented in Definition 4, i.e. the strong or weak auxiliary modulus has to be bounded on the system trajectories, while a local auxiliary modulus can be just a sufficiently slowly growing function of time.

Using the inequality (17) the following corollary on Lemma 3 can be given.

Corollary 2 Consider the system (1) having UO. Let there exist a smooth Lyapunov function $V: \mathbb{R}^{n} \rightarrow \mathbb{R}_{\geq 0}$ as in Lemma 3. The system (1) is OFTS if for any $\varepsilon \in \mathbb{R}_{\geq 0}$ there exists $T_{\varepsilon} \in \mathbb{R}_{\geq 0}$ such that

$$
\int_{0}^{\alpha_{2} \circ \gamma(\varepsilon)} \frac{d s}{\kappa_{1}(s)} \leq \frac{T_{\varepsilon}}{1+\kappa_{2}\left(\varrho\left(\varepsilon, T_{\varepsilon}\right)\right)}<+\infty,
$$

where $\kappa_{1}, \kappa_{2} \in \mathcal{K}$ as in (17). Moreover, $T\left(x_{0}\right) \leq T_{\varepsilon}$ for $\left\{x_{0} \in \mathbb{R}^{n}:\left\|x_{0}\right\| \leq \varepsilon\right\}$.

Proof. The proof is a direct consequence of Lemma 3 and the inequality (17). Indeed, let the inequality (23) is satisfied, then we have

$$
\begin{gathered}
T\left(x_{0}\right) \leq \int_{0}^{\alpha_{2}(\varepsilon)} \frac{d s}{\alpha_{3}\left(s, \varrho\left(\varepsilon, T_{\varepsilon}\right)\right)} \\
\leq\left(1+\kappa_{2}\left(\varrho\left(\varepsilon, T_{\varepsilon}\right)\right)\right) \int_{0}^{\alpha_{2}(\varepsilon)} \frac{d s}{\kappa_{1}(s)} \leq T_{\varepsilon}<+\infty .
\end{gathered}
$$

Remark 2 If the presented conditions are satisfied for the set $\left\{x_{0} \in \mathbb{R}^{n}: V\left(x_{0}\right) \leq c\right\}, c \in \mathbb{R}_{>0}$, then the system (1) is locally OFTS.

Example 1 The two tank hydraulic system with controlled tap is depicted in Fig. 3. The system can be presented as

$$
\dot{x}_{1}=-\frac{a_{1}}{S_{t 1}} x_{1}^{0.5} g(x), \quad \dot{x}_{2}=\frac{a_{1}}{S_{t 1}} x_{1}^{0.5} g(x)+\frac{1}{S_{t 2}} u-\frac{a_{2}}{S_{t 2}} x_{2}^{0.5},
$$

where the liquid levels in tanks represent the state vector $x=\left(x_{1}, x_{2}\right)^{T}=\left(L_{1}, L_{2}\right)^{T} \in \mathbb{R}_{>0}^{2}, u \in \mathbb{R}_{\geq 0}$ is the input, $g(x) \in[0,1]$ is the tap control function, $S_{t 1}, S_{t 2}$ denote the cross sections of the tanks and $a_{1}, a_{2}$ correspond to the flow rates. Let $a_{2}=0, a_{1}=S_{t 1}=u=1$ with appropriate units and the output is $y=x_{1}$.

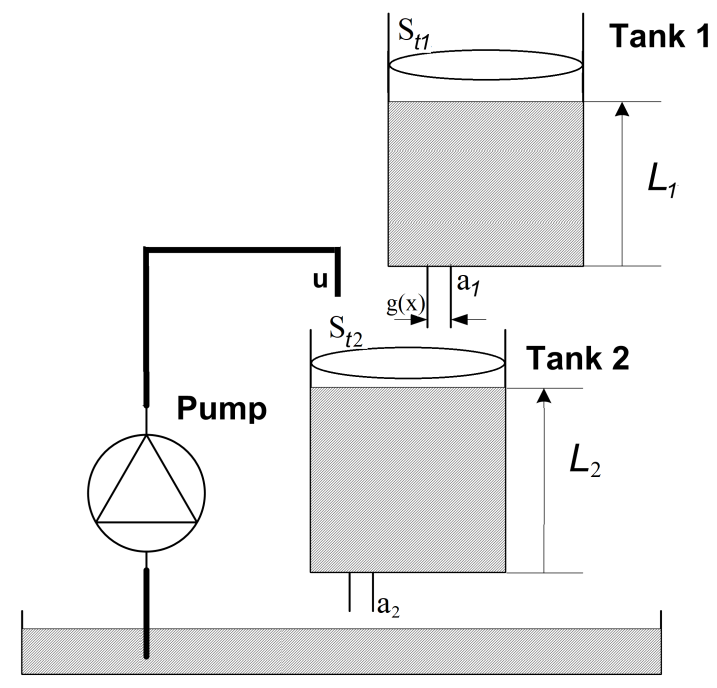

Fig. 1. Two tank hydraulic system

I. Let $g(x)=\frac{1}{1+x_{2}}$. Choose $V(x)=x_{1}^{1.5}$. The function $V$ satisfies (9) and (6) with $\lambda(x)=x_{2}$ since

$$
\dot{V}(x)=-1.5 \frac{V^{2 / 3}(x)}{1+x_{2}}=-1.5 \frac{V^{2 / 3}(x)}{1+\lambda(x)}=-\alpha_{3}(V(x), \lambda(x))
$$

and (9) is satisfied for $\alpha_{3}(s, r)=1.5 \frac{\mathrm{s}^{2 / 3}}{1+r}$. Since $x_{2}(t) \leq$ $x_{1}(0)+x_{2}(0)+\frac{1}{S_{t 2}} t$ we have $\lambda(x) \leq x_{1}(0)+x_{2}(0)+$ $\frac{1}{S_{t 2}} T=\varrho(x(0), T)$ for $t \in[0, T]$. Then, considering (20) we obtain the inequality $2 \sqrt{\varepsilon}\left(1+\varrho\left(x(0), T_{\varepsilon}\right)\right) \leq T_{\varepsilon}$, that has a solution for $\varepsilon<0.25 S_{t 2}^{2}$, i.e. the system is locally OFTS by Corollary 2 .

II. Let $g(x)=\frac{1}{1+x_{2}^{0.5}}$. Then, for $V=x_{1}^{1.5}$ one can obtain the inequality $2 \sqrt{\varepsilon}\left(1+\varrho\left(x(0), T_{\varepsilon}\right)^{0.5}\right) \leq T_{\varepsilon}$, that always has a solution. Thus, by Corollary 2 the system is globally OFTS. The results of simulation are shown in Fig. 2 for $x(0)=(10,2)^{T}$ using the logarithmic scale in order to demonstrate finite-time convergence rate of the output.

Note, that in this example $\lambda(x)$ is a local auxiliary modulus, that is a sufficiently slowly growing function of time.

Remark 3 Note that the presented results can be used for some systems with oGAS-Lyapunov function. For example, considering the system (24) with $y=\sin x_{1}$ the function $V=\left|x_{1}\right|^{1.5}$ is not OLoGAS-Lyapunov function since (9) is not satisfied. However, due to the output 


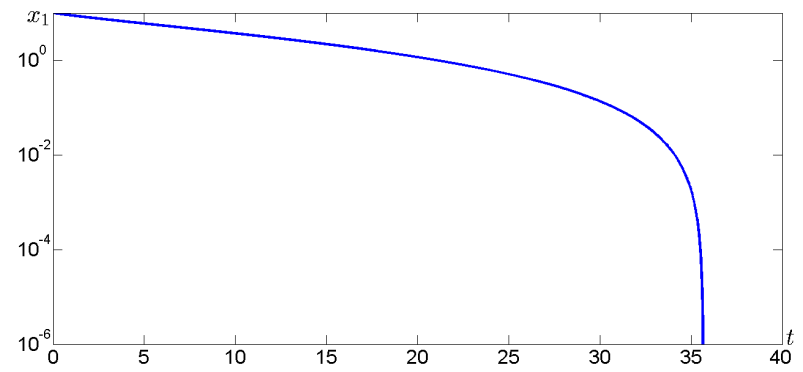

Fig. 2. Output variable $y=x_{1}$ of two tank hydraulic system $y=x_{1}$ is FTS (see Example 1 ) it is obvious that the system with $y=\sin x_{1}$ is also OFTS.

It is worth to highlight that in the presented results the settling-time function might be discontinuous. In [26] it is shown that for the case of state finite-time convergence the settling-time function $T(x)$ is continuous if and only if it is continuous at 0 . It is not satisfied for output stability in general case as it is shown in the following example.

Example 2 Consider the system

$$
\dot{x}_{1}=-\frac{\left\lfloor x_{1}\right\rceil^{0.5}}{1+\left|x_{2}\right|}, \quad \dot{x}_{2}=-x_{2} .
$$

I. Let $y=x_{1}$. The system is OFTS (using Theorem 2 with weak auxiliary modulus $\left.\lambda=x_{2}\right)$ and $T\left(x_{0}\right)=$ $\ln \left(\left(\left|x_{2}(0)\right|+1\right) e^{2 \sqrt{\left|x_{1}(0)\right|}}-\left|x_{2}(0)\right|\right)$ is continuous.

II. Let $y=x_{1} x_{2}$. Since $x_{2}(t)=0$ only if $x_{2}(0)=0$, then the system is OFTS with

$$
T\left(x_{0}\right)=\ln \left(\left(\left|x_{2}(0)\right|+1\right) e^{2 \sqrt{\left|x_{1}(0)\right|}}-\left|x_{2}(0)\right|\right)
$$

for $x_{2}(0) \neq 0$ and $T\left(x_{0}\right)=0$ for $x_{2}(0)=0$, i.e. the settlingtime function is discontinuous at $\left(x_{1}, 0\right), x_{1} \in \mathbb{R} \backslash\{0\}$.

The paper [18] presents the necessary and sufficient conditions for continuity of the settling-time function. The following proposition extends this result for the case of output stability.

\section{Proposition 1}

(i) If $x_{0} \in \mathbb{R}^{n}$ and $t \in \mathbb{R}_{\geq 0}$, then

$$
T\left(X\left(t, x_{0}\right)\right)=\max \left\{T\left(x_{0}\right)-t, 0\right\} .
$$

(ii) $T$ is continuous on $\mathbb{R}^{n}$ if and only if $T$ is continuous on $\mathcal{Y}$.

Proof. (i) According to (12) we have $T\left(X\left(t, x_{0}\right)\right)=$ $\inf \left\{T_{0} \geq 0: Y\left(t+\tau, x_{0}\right)=0 \forall \tau \geq T_{0}\right\}$. On other hand, $T\left(x_{0}\right)=\inf \left\{T_{0} \geq 0: Y\left(t+\tau, x_{0}\right)=0 \forall(t+\tau) \geq T_{0}\right\}$. Finally applying (13) we obtain (25). (ii) Necessity is immediate. To prove sufficiency, suppose that $T$ is continuous on $\mathcal{Y}$.

Let $z \in \mathbb{R}^{n}$ and consider a sequence $\left\{z_{m}\right\}$ in $\mathbb{R}^{n}$ such that $z_{m}$ converges to $z$. Let $\tau^{-}=\liminf _{m \rightarrow \infty} T\left(z_{m}\right)$ and $\tau^{+}=\limsup _{m \rightarrow \infty} T\left(z_{m}\right)$. Note that both $\tau^{-}$and $\tau^{+}$are in $\mathbb{R}_{\geq 0} \cup\{+\infty\}$ and

$$
\tau^{-} \leq \tau^{+}
$$

Next, let $\left\{z_{l}^{+}\right\}$be a subsequence of $\left\{z_{m}\right\}$ such that $T\left(z_{l}^{+}\right) \rightarrow \tau^{+}$as $l \rightarrow \infty$. The sequence $\left\{\left(T(z), z_{l}^{+}\right)\right\}$ converges in $\mathbb{R}_{\geq 0} \times \mathbb{R}^{n}$ to $(T(z), z)$. By continuity, $X\left(T(z), z_{l}^{+}\right) \rightarrow X(T(z), z)$, and by equation (13), $Y\left(T(z), z_{l}^{+}\right) \rightarrow Y(T(z), z)=0$ as $l \rightarrow \infty$. Since $T$ is assumed to be continuous at $z \in \mathcal{Y}$, then $T\left(X\left(T(z), z_{l}^{+}\right)\right) \rightarrow T(z)=0$ as $l \rightarrow \infty$. Using (25) with $t=T(z)$ and $x_{0}=z_{l}^{+}$, we obtain $\max \left\{T\left(z_{l}^{+}\right)-T(z), 0\right\} \rightarrow$ 0 as $l \rightarrow \infty$. Thus $\max \left\{\tau^{+}-T(z), 0\right\}=0$, that is,

$$
\tau^{+} \leq T(z)
$$

Now, let $\left\{z_{l}^{-}\right\}$be a subsequence of $\left\{z_{m}\right\}$ such that $T\left(z_{l}^{-}\right) \rightarrow \tau^{-}$as $l \rightarrow \infty$. It follows from (26) and (27) that $\tau^{-} \in \mathbb{R}_{\geq 0}$. Therefore, the sequence $\left\{\left(T\left(z_{l}^{-}\right), z_{l}^{-}\right)\right\}$ converges in $\overline{\mathbb{R}}_{\geq 0} \times \mathbb{R}^{n}$ to $\left(\tau^{-}, z\right)$. Since $Y$ is continuous, it follows that $Y\left(T\left(z_{l}^{-}\right), z_{l}^{-}\right) \rightarrow Y\left(\tau^{-}, z\right)$ as $l \rightarrow \infty$. Equation (13) implies that $Y\left(T\left(z_{l}^{-}\right), z_{l}^{-}\right)=0$ for each $l$. Hence $Y\left(\tau^{-}, z\right)=0$ and according to settling-time function definition

$$
T(z) \leq \tau^{-}
$$

From (26), (27), and (28) we conclude that $\tau^{-}=\tau^{+}=$ $T(z)$ and hence $T\left(z_{m}\right) \rightarrow T(z)$ as $m \rightarrow \infty$.

The next results are presented for the SIoGAS class of systems.

Corollary 3 Consider the SIoGAS system (1). The following properties are equivalent:

(i) the system (1) is OFTS with a continuous settling-time function;

(ii) there exists a real number $c>0, \mu \in(0,1)$ and $a$ Lyapunov function $V: \mathbb{R}^{n} \rightarrow \mathbb{R}_{\geq 0}$ satisfying (9) and

$$
\dot{V}(x) \leq-c V(x)^{\mu}
$$

for all $x \in \mathbb{R}^{n}$. Moreover, the settling-time function satisfies $T(x) \leq \frac{1}{c(1-\mu)} V(x)^{1-\mu}$.

Proof. $(i) \Rightarrow($ ii $)$ Define $V: \mathbb{R}^{n} \rightarrow \mathbb{R}_{\geq 0}$ by $V(x)=$ $(T(x))^{\frac{1}{1-\mu}}$. Then $V$ is continuous and positive definite and, for $x \in \mathcal{Y}$ we have $\dot{V}(x)=0$ due to (9) and (13). 
For $x \in \mathbb{R}^{n} \backslash \mathcal{Y}(25)$ implies that $V(x(t))$ is continuously differentiable on $[0, T(x))$ so that

$$
\dot{V}(x)=-\frac{1}{1-\mu}(T(x))^{\frac{\mu}{1-\mu}}=-\frac{1}{1-\mu}(V(x))^{\mu} .
$$

Thus $\dot{V}$ is real valued, continuous, and negative definite on $\mathbb{R}^{n} \backslash \mathcal{Y}$ and satisfies $\dot{V}(x)+c(V(x))^{\mu}=0$ for all $x \in \mathbb{R}^{n}$ with $c=\frac{1}{1-\mu}$.

$($ ii $) \Rightarrow(i)$ The sufficiency of (29) can be checked by integration:

$$
\begin{aligned}
T(x)= & \int_{0}^{V(x)} \frac{d s}{-\dot{V}\left(X\left(\theta_{x}(s), x\right)\right)} \leq \int_{0}^{V(x)} \frac{d s}{c V\left(X\left(\theta_{x}(s), x\right)\right)^{\mu}} \\
& =\int_{0}^{V(x)} \frac{d s}{c s^{\mu}}=\frac{1}{c(1-\mu)} V(x)^{1-\mu}<+\infty
\end{aligned}
$$

Due to $(30), T(z) \leq \frac{1}{c(1-\mu)} \alpha_{2}(\|h(z)\|)^{1-\mu}$ that by continuity of $\alpha_{2}$ implies continuity of $T$ at $\mathcal{Y}$. Then by Proposition 1 the settling-time function is continuous.

Example 3 Consider the system presented in Example 1. Let $g(x)=0.5+0.25 \sin \left(x_{1} x_{2}\right)$. Let $V=x_{1}^{1.5}$. The function $V$ is SIoGAS-Lyapunov function due to $\dot{V}(x)=-1.5 x_{1}\left(0.5+0.25 \sin \left(x_{1} x_{2}\right)\right) \leq-0.375 V^{2 / 3}$. Then the system is globally OFTS due to Corollary 3 and $T\left(x_{0}\right) \leq 8 V\left(x_{0}\right)^{1 / 3}=8 \sqrt{x_{1}(0)}$. The results of simulation are shown in Fig. 3 for $x(0)=(1,0)^{T}$.

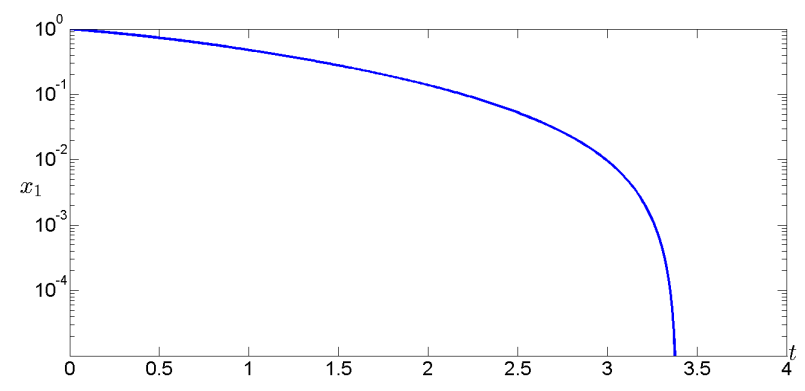

Fig. 3. Output variable $y=x_{1}$ of two tank hydraulic system

\section{Conclusions}

In the paper necessary and sufficient Lyapunov characterizations of output finite-time stability are presented for the class of OLoGAS and SIoGAS systems (1). The presented stability analysis of OFTS opens a lot of topics for future research. The main of them are: extension to a wider class of systems (e.g. with external inputs); extension for output fixed-time stability (a special case of OFTS with a globally bounded settling-time function); application of the results for control (e.g. output regulation, adaptive control, trajectory tracking control, etc.) and observer design problems.

\section{References}

[1] K. Zimenko, D. Efimov, A. Polyakov, A. Kremlev. On Notions of Output Finite-Time Stability. European Control Conference (ECC), Naples, 2019.

[2] H.G. Kankanamalage, Y. Lin, Y. Wang. On notions of inputto-output stability for nonlinear systems with time delays. Proc. of IEEE International Conference on Control and Automation, pages 490-495, 2017.

[3] G. Wang, X. Zhang, S. Mei, S. Wu. Input-to-output stability analysis of interconnecting microgrids. Proc. of 4th International Conference on Electric Utility Deregulation and Restructuring and Power Technologies, pages 1097-1103, 2011.

[4] H.G. Kankanamalage, Y. Wang. Lyapunov descriptions of robust output stability for systems with delays. Proc. of 55th Conference on Decision and Control, pages 6416-6421, 2016.

[5] R.G. Sanfelice. Results on input-to-output and inputoutput-to-state stability for hybrid systems and their interconnections. Proc. of the IEEE Conference on Decision and Control, pages 2396-2401, 2010.

[6] D. Efimov, A. Loria, E. Panteley. Robust output stabilization: Improving performance via supervisory control. International Journal of Robust and Nonlinear Control, vol.21(10), pages 1219-1236, 2011.

[7] A.L. Fradkov, A.Yu. Pogromsky. Introduction to oscillations and chaos. World Scientific, Singapore, 1998.

[8] J. Liu, M. French. Robust stability of input-output systems with initial conditions. SIAM Journal on Control and Optimization, vol. 53(3), pages 1625-1653, 2015.

[9] V.I. Vorotnikov, Yu.G. Martyshenko. On partial stability theory of nonlinear dynamic systems. Journal of Computer and Systems Sciences International, vol. 49(5), pages 702709, 2010.

[10] C. Jammazi. Continuous and discontinuous homogeneous feedbacks finite-time partially stabilizing controllable multichained systems. SIAM Journal on Control and Optimization, vol. 52(1), pages 520-544, 2014.

[11] V. Vorotnikov. Partial stability, stabilization and control: some recent results. in 15th IFAC Triennial World Congress, 2002.

[12] V.V. Rumyantsev, A.S. Oziraner. Stability and stabilization of motion with respect to part of variables. Nauka, Moscow, 1987.

[13] A.L. Fradkov, I.V. Miroshnik, V.O. Nikiforov. Nonlinear and adaptive control of complex systems. Kluwer, 1999.

[14] K.S. Narendra, A.M. Annaswamy. Stable adaptive systems. Prentice-Hall, Inc. Upper Saddle River, NJ, 1989.

[15] M. Arcak and P. Kokotović. Nonlinear observers: a circle criterion design and robustness analysis. Automatica, vol. 37(12), pp. 1923-1930, 2001.

[16] V. Andrieu, S. Tarbouriech. LMI Sufficient conditions for contraction and synchronization. 11th IFAC Symposium for Nonlinear Control, Vienna, 2019.

[17] Z. Hai-Ping, M. Feng-Xiang. On the stability of nonholonomic mechanical systems with respect to partial variables. Appl.Math. Mech, vol. 16(3), pp. 237-245, 1995.

[18] W.M. Haddad, A. L'Afflitto. Finite-time partial stability and stabilization, and optimal feedback control. Journal of the Franklin Institute, vol. 352, pages 2329-2357, 2015. 
[19] C. Jammazi. On a sufficient condition for finite-time partial stability and stabilization: applications. IMA Journal of Mathematical Control and Information, vol. 27(1), pages 2956,2010 .

[20] D. Angeli, E. Sontag. Forward completeness, unboundedness observability, and their Lyapynov characterizations. Systems \& Control Letters, vol. 38, pages 209-217, 1999.

[21] S.N. Dashkovskiy, D.V. Efimov, E.D. Sontag. Input to State Stability and Allied System Properties. Automation and Remote Control, vol. 72, no. 8, pages 1579-1614, 2011.

[22] B. Ingalls, Y. Wang. On Input-to-Output Stability for Systems not Uniformly Bounded. Proc. NOLCOS, St. Petersburg, July 2001.

[23] E.D. Sontag, Y. Wang. Lyapunov Characterizations of Input to Output Stability. SIAM J. Control Optim., no. 39(1), pages 226-249, 2000.

[24] E.D. Sontag, Y. Wang. A Notion of Input to Output Stability. Proc. Eur. Control Conf., Brussels, July 1997, DOI: 10.23919/ECC.1997.7082720.

[25] E.D. Sontag, Y. Wang. Notions of Input to Output Stability. Syst. Control Lett., no. 38(4-5), pages 235-248, 1999.

[26] S.P. Bhat, D.S. Bernstein. Finite-time stability of continuous autonomous systems. SIAM J. Control Optim., vol. 38, no. 3, pages 751-766, 2000.

[27] F. Amato, R. Ambrosino, C. Cosentino, G. De Tommasi. Input-output finite time stabilization of linear systems. Automatica, vol. 46(9), pages 1558-1562, 2010.

[28] A.L. Fradkov, I.V. Miroshnik, V.O. Nikiforov. Nonlinear and Adaptive Control of Complex Systems. Dordrecht: Kluwer Acad. Publ., 528 pages, 1999.

[29] R. Engel, G. Kreisselmeier. A continuous-time observer which converges in finite time. IEEE Transactions on Automatic Control, vol. 47, pages 1202-1204, 2002.

[30] W. Perruquetti, T. Floquet, E. Moulay. Finite-time observers: Application to secure communication. IEEE Transactions on Automatic Control, vol. 53(1), pages 356$360,2008$.

[31] I. Karafyllis, Z.P. Jiang. Stability and Stabilization of Nonlinear Systems. Communications and Control Engineering, Springer-Verlag, London, 2011.

[32] E. Sontag. Comments on integral variants of input-to-state stability. Systems \& Control Letters, vol. 34, pages 91-100, 1998.

[33] Y. Lin, E.D. Sontag, Y. Wang. A smooth converse Lyapunov theorem for robust stability. SIAM Journal on Control and Optimization, vol. 34(1), pages 124-160, 1996.

[34] K. Zimenko, D. Efimov, A. Polyakov. On Condition for Output Finite-Time Stability and Adaptive Finite-Time Control Scheme. Proc. 58th IEEE Conference on Decision and Control, Nice, 2019.

[35] E. Guzman, J. Moreno. New finite-time convergent and robust direct model reference adaptive control for SISO linear time invariant systems. In 50th IEEE Conference on Decision and Control, Florida, USA, pp. 70277032, 2011. 